\title{
Stellar Convection as a Low Prandtl Number Flow
}

\author{
Josep M. Massaguer \\ Departament de Física Aplicada Universitat Politècnica de Catalunya, \\ 08034 Barcelona, Spain
}

\begin{abstract}
Thermal convection in the Sun and cool stars is often modeled with the assumption of an effective Prandtl number $\sigma \simeq 1$. Such a parameterization results in masking of the presence of internal shear layers which, for small $\sigma$, might control the large scale dynamics. In this paper we discuss the relevance of such layers in turbulent convection. Implications for heat transport - i.e. for the Nusselt number power law - are also discussed.
\end{abstract}

\section{Introduction}

Astrophysical plasmas are almost inviscid fluids. In stellar convection zones these fluids are optically thick almost everywhere, and radiative heat transport is there an efficient diffusion process. Therefore, thermal convection in stars is a low Prandtl number flow, with the Prandtl number $\sigma=\nu / \kappa$ defined as the ratio of the viscous, $\nu$, and thermal, $\kappa$, molecular diffusion coefficients. In the solar case, for instance, the molecular viscosity is so small compared to the thermal diffusion that $\sigma$ can reach values of $\sigma=10^{-9}$, meaning that viscous dissipation is only relevant for layers of several centimeters thick. Nobody can expect such small Prandtl numbers to be of any relevance for describing the large scale dynamics of the solar convection zone, but viscous dissipation cannot simply be neglected as it will break the energy balance. Therefore, the real question is about modeling the viscous diffusion term or, stated otherwise, a parameterization for $\sigma$.

The relevance of the Prandtl number in fully developed turbulent convection has been widely questioned in the past - see, for instance, the footnote on page 424 of Monin and Yaglom (1971). At large scales, molecular diffusion is negligible as compared to turbulent diffusion, so it might be plausible to describe the large scale dynamics with no reference to the molecular diffusion coefficients, not even to their ratio, the Prandtl number. However the implication is not that obvious. Molecular coefficients can play a role in building up small scale structures, mostly in internal boundary layers, delimiting convective eddies, thermals or plumes. 


\section{On the effective Prandtl number}

The most popular parameterization technique is to take instead of the molecular value its turbulent analogue, the turbulent Prandtl number $\sigma_{t}=\nu_{t} / \kappa_{t}$, where $\nu_{t}$ and $\kappa_{t}$ are eddy transport coefficients for momentum and heat. A proper definition of the scalar quantities $\nu_{t}$ and $\kappa_{t}$ can only be made for isotropic turbulence or for some prescribed geometries - free shear flows, near wall turbulence, axisymmetric jets, etc. - where the eddy coefficients have a very precise meaning; otherwise $\nu_{t}$ and $\kappa_{t}$ become tensorial quantities. From a large amount of measurements for different flows and geometries the conclusion might be that $\sigma_{t}$ takes a value close to one, with the most frequent values in the range $\sigma_{t}=0.6-0.9$. See Launder (1978) for an authoritative review.

It is thought that taking $\sigma \simeq 1$ instead of the true $\sigma$ in the Navier-Stokes equation is a safe procedure for describing the large scale dynamics, but it is not. Two flows computed with different $\sigma$ values may show the same $\sigma_{t}$ value but different large scale dynamics. The relevant question to ask is about the existence of an asymptotic value $\sigma_{a s}$ such that computing the flows for any $\sigma<\sigma_{a s}$, everything else being unchanged, produces indistinguishable large scale dynamics. So far the question is still open, though existing numerical work puts an upper bound $\sigma_{a s}<$ 0.1 to this asymptotic value. In Massaguer and Mercader (1988) and Massaguer et al. (1990) this asymptotic limit is conjectured from the onset of an instability to be described below. In Toomre et al. (1990) it has been found, for highly stratified fluids, that changing the Prandtl number in the range $\sigma=0.1-1$ results in a significant change in the size of the observed structures. And the latter authors also report, from an unpublished work by J. Herring that, even in the Boussinesq convection, changing $\sigma$ in that range produces significant changes in the large scale structure. Neither of the previously quoted works is conclusive, and indeed the whole subject is still open. But it is remarkable that Kraichnan (1962), from a discussion based on a mixing-length treatment, conjectured for the Prandtl number of the transition between high and low Prandtl number regimes a value that he estimates "with low confidence as about 0.1". His model is based on a detailed study of the thermal and viscous boundary layers, and the estimate depends on the value of the ratio $R e_{T} / P e_{T}$ between the Reynolds and Pèclet numbers at the edge of the thermal layer. But he warns the reader that "if the value turns out to be greater than the nominal value 10 which we have used, the effect of the theory would be to lower the value of $\sigma$ which separates the low and high Prandtl number ranges... values of $R e_{T} / P e_{T}$ greater than 10 would not be surprising".

Such a low separating value of $\sigma$ will originate in the turbulent shear boundary layers existing at the edge of the big eddies, where large scale isotropy and homogeneity will break down. In order to quantify such an effect, we can borrow some ideas from subgrid scale modeling. As discussed, for instance, by Lessieur (1987) in a chapter on Large Eddy Simulation, the contribution to the large scale dynamics by modes with wavenumbers $k>k_{c}$, with $k_{c}$ in the inertial range, can be described by the eddy coefficients 


$$
\begin{aligned}
& \nu_{t}\left(k_{c}\right)=0.28 \sqrt{\frac{E\left(k_{c}\right)}{k_{c}}} \\
& \kappa_{t}\left(k_{c}\right)=0.42 \sqrt{\frac{E\left(k_{c}\right)}{k_{c}}}
\end{aligned}
$$

thus giving $\sigma_{t} \simeq 0.67$. But the Prandtl number entering the equations is $\sigma=$ $\left(\nu+\nu_{t}\right) /\left(\kappa+\kappa_{t}\right)$. In the limit of small $\sigma$ this expression can be approximated as

$$
\sigma\left(k_{c}\right) \simeq \frac{0.28 P e\left(k_{c}\right)}{1+0.42 P e\left(k_{c}\right)}
$$

where $P e=v \ell / \kappa$ is a Pèclet number defined by $v=\sqrt{k_{c} E\left(k_{c}\right)}$ and $\ell=k_{c}^{-1}$ and $k_{c}$ is a wavenumber in the inertial range. Shear layers put a strong constraint on the value of $k_{c}$. Following a suggestion from Kraichnan's paper, we can define the edge of one such layer as a point where diffusion and advection of heat take the same value, implying $P e\left(k_{c}\right)=0.5$ and $\sigma\left(k_{c}\right) \simeq 0.1$ in agreement with papers quoted above. The asymptotic value $\sigma_{a s}$ corresponds to the maximum value of $k_{c}$.

\section{Shear dynamics}

Shear layers can modify scalings substantially, but including them in the balances is a hard task. In order to explain where difficulties lie, let us consider thermal convection in a Boussinesq fluid. The velocity $v$ is a solenoidal field, and can be split into a poloidal $v_{P}=\nabla \times \nabla \times\left(\phi e_{z}\right)$ and a toroidal $v_{T}=\nabla \times\left(\psi e_{z}\right)$ component, $v=v_{P}+v_{T}$, where $e_{z}$ is a unit vector antiparallel to gravity and the scalars $\phi$ and $\psi$ are associated to the vertical components of the velocity and vorticity fields. As a physical picture for the poloidal velocity field we can think of a vertical disk rotating around its horizontal axis. With a string of such a disks we can reproduce the flow inside rolls and hexagons, the best known convective structures. Toroidal flows can be represented by horizontal disks rotating around their vertical axis. In most contexts left-handed and right-handed toroidal flows are required so as to cancel the vertical component of the angular momentum and, depending on the geometry of the container, vertically rotating disks will be paired off either horizontally or vertically. A final patchwork with such pairs will always result in important shear stresses A crucial step in understanding shear processes in thermal convection is to realize that, besides boundary effects, thermal convection can always be modeled as a poloidal flow. The poloidal flow is always an exact solution of the Navier-Stokes equation, though it may be unstable. Most known scalings, with Kraichnan's exception, reflect global balances for the poloidal component. A recent attempt by Castaing et al. (1989) to include shear stresses is also to be mentioned.

The poloidal velocity field scales, roughly speaking, as $v_{P} \simeq \kappa / \ell$ and the Reynolds number takes a value $R e=v_{P} \ell / \kappa \simeq \sigma^{-1}$. Decreasing $\sigma$ increases $R e$ without bound, and at low $\sigma$ any poloidal field is expected to be shear unstable. 
The onset of this shear instability has been examined in Massaguer and Mercader (1988) and an asymptotic regime for small $\sigma$ has been found. It is also worth mentioning that near the onset of instability the flow turns out to be chaotic with components $v_{P}$ and $v_{T}$ uncorrelated (Massaguer et al., 1990). Therefore, the flow is chaotic in time and intermittent in space. The toroidal velocity field arises as a secondary flow, and its description requires a previous knowledge of the nonlinear dynamics of the poloidal flow, mostly of its geometry. So it is not surprising that most early attempts at modeling turbulent convection simply neglected the contribution of the toroidal component. In Malkus' theory of turbulence, for instance, - see Spiegel's (1962) reformulation - the vertical vorticity component is treated as a damped mode, implying $\boldsymbol{v}_{T}=0$. A similar difficulty arises in Canuto et al. (1987) extension of Ledoux, Schwarzchild \& Spiegel's spectral model. A preliminary attempt to include secondary instabilities can be found in Canuto et al. (1990).

\subsection{Dimensional analysis}

Introducing a scale of inhomogeneity $\ell=k_{c}^{-1}$ associated with the internal boundary layers, as we have done before, results in an additional degree of freedom in the well known dimensional analysis - see Massaguer (1990) and references therein. It is widely agreed that at low $\sigma$ values the large scale dynamics should be independent of the molecular viscosity $\nu$. And the Nusselt number $N$-i.e.: the non-dimensional heat flux - becomes $N=N(R a \sigma, \ell / d)$, where $R a$ is the Rayleigh number and $d$ is the layer thickness. For an homogeneous and isotropic flow $\ell / d \simeq 1$, the molecular diffusion of heat should be negligible, $\kappa<<\kappa_{t}\left(k_{c}\right)$, and the the heat flux cannot depend on $\kappa$. The only possible power-law for $N$ is the classical mixing-length scaling $N \propto(R a \sigma)^{1 / 2}$.

If thin internal boundary layers are present in the flow, $\ell / d<<1$, the heat flux will be partially controlled by them. Let us assume the extreme situation where the flux is completely independent of the top and bottom wall boundaries. If the temperature gradient is $\partial_{z} T$, the gravity $g$ and the expansion coefficient $\alpha$,

$$
R a \sigma=\frac{\alpha g d^{4} \partial_{z} T}{\kappa^{2}}
$$

then the only power-law for $N$ independent of $d$-i.e. controlled by $\ell$-is $N \propto$ $\ell / d(R a \sigma)^{1 / 4}$. At this point we must recall that for large $\sigma$ values the heat flux behaves as $N \propto R a^{1 / 3}$, which corresponds to a flow controlled by wall boundary layers; and also, that recent experiments with gaseous helium $(\sigma \simeq 1)$ by Threlfall (1975), and Castaing et al. (1989) give $N \propto R a^{2 / 7}$, which corresponds, according to the scaling done by the latter authors, to a shared control of both internal and wall boundary layers. The increasing value of the exponents $1 / 4<2 / 7<1 / 3$ corresponds to an increasing dominance of the wall boundary layers with respect to internal boundary layers - please, notice that $2 / 7$ is the simplest integer ratio between $1 / 4$ and $1 / 3$.

The lack of well defined criteria for choosing the exponent $n$ in the expression $N \propto(R a \sigma)^{n}$ is a real shortcoming for stellar convection. The above discussion 
shows $n$ to be in the range $n=0.25-0.50$, with some preference for the smaller values, but the physics depends on the evaluation of very subtle balances, and detailed modeling is required.

\subsection{A final remark}

Shear layers are induced by the presence of a toroidal component in the turbulent flow, and this may also result in the enhancement of the horizontal velocity components of the Reynolds stresses. Clear indications of non-equipartition of energy between the poloidal and toroidal velocity components have been reported by Massaguer et al. (1990). Such a large scale anisotropic transport has been invoked a number of times as a mechanism for redistributing angular momentum on the solar surface, mostly in the context of the differential rotation but, to the best of our knowledge, low $\sigma$ modeling has not even been attempted.

Acknowledgements. The present work was supported by the Dirección General de Investigación Científica y Técnica (DGICYT) under grant PS87-0107. A travel grant from CIRIT is also acknowledged.

\section{References}

Canuto, V.M., Goldman, I., Chasnov, J.: 1987, Phys. Fluids 30, 3391

Canuto, V.M., Cheng, Y., Hartke, G.J., Schilling, O., Chasnov, J.: 1990, J. Fluid Mech. (to be published)

Castaing, B., Gunaratne, G., Heslot, F.L., Kadanoff, L., Libchaber, A., Thomae, S., Wu, X.Z., Zaleski, S., Zanetti, G.: 1989, J. Fluid Mech. 204, 1

Kraichnan, R.H.: 1962, Phys. Fluids 5, 1374

Launder, B.E.: 1978, in "Turbulence", ed. P. Bradshaw, p. 231, Topics in Applied Physics 12, Springer

Lessieur, M.: 1987, Turbulence in Fluids, M. Nijhoff Publ., Dordrecht

Massaguer, J.M.: 1990, in "Inside the Sun", eds. G. Berthomieu and M. Cribier p. 101, Kluwer.

Massaguer, J.M., Mercader, I.: 1988, J. Fluid Mech. 189, 367

Massaguer, J.M., Mercader, I., Net, M.: 1990, J. Fluid Mech. 214, 579

Monin, A.S., Yaglom, A.M.: 1971, Statistical Fluid Mechanics, vol 1, The MIT Press.

Spiegel, E.A.: 1962, in "Mécanique de la Turbulence", Colloques Internationaux du CNRS à Marseille (Editions CNRS Paris). p. 181

Threlfall, D.C.: 1975, J. Fluid Mech. 67, 17

Toomre, J., Brummell, N., Cattaneo, F., Hurlburt, N.E.: 1990, Computer Phys. Commun. $\mathbf{5 0}, 105$ 